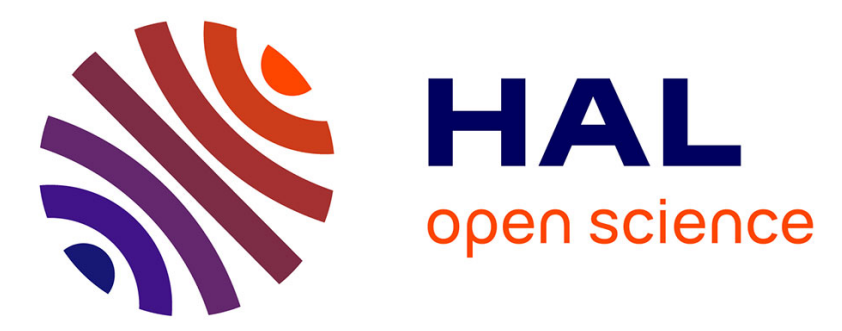

\title{
The neural substrates of musical memory revealed by fMRI and two semantic tasks.
}

\author{
Mathilde Groussard, Géraldine Rauchs, Brigitte Landeau, Fausto Viader, \\ Béatrice Desgranges, Francis Eustache, Hervé Platel
}

\section{- To cite this version:}

Mathilde Groussard, Géraldine Rauchs, Brigitte Landeau, Fausto Viader, Béatrice Desgranges, et al.. The neural substrates of musical memory revealed by fMRI and two semantic tasks.: Neural substrates of musical memory. NeuroImage, 2010, 53 (4), pp.1301-9. 10.1016/j.neuroimage.2010.07.013 . inserm-00538633

\section{HAL Id: inserm-00538633 https://www.hal.inserm.fr/inserm-00538633}

Submitted on 23 Nov 2010

HAL is a multi-disciplinary open access archive for the deposit and dissemination of scientific research documents, whether they are published or not. The documents may come from teaching and research institutions in France or abroad, or from public or private research centers.
L'archive ouverte pluridisciplinaire HAL, est destinée au dépôt et à la diffusion de documents scientifiques de niveau recherche, publiés ou non, émanant des établissements d'enseignement et de recherche français ou étrangers, des laboratoires publics ou privés. 


\title{
The neural substrates of musical memory revealed by fMRI and two semantic tasks
}

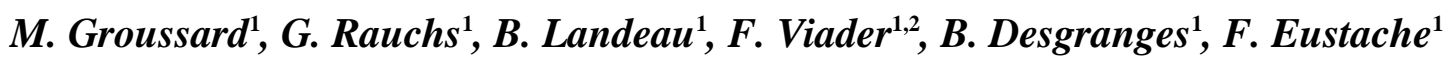 \\ \& H. Platel ${ }^{1}$
}

Brief running title: Neural substrates of musical memory

\begin{abstract}
${ }^{1}$ Inserm-EPHE-Université de Caen/ Basse-Normandie, Unité U923, GIP Cyceron, CHU Côte de Nacre, Caen, France.
\end{abstract}

${ }^{2}$ Département de Neurologie, CHU Côte de Nacre, Caen, France

Correspondence and reprint requests:

Hervé Platel, Inserm - EPHE-Université de Caen/Basse-Normandie, Unité U923,

U.F.R de Psychologie, Université de Caen/Basse-Normandie, Esplanade de la Paix, 14032 Caen Cedex, France

Phone: +33 (0)2 315665 91; Fax: +33 (0)2 315666 93, e-mail: herve.platel@unicaen.fr 


\begin{abstract}
Recognizing a musical excerpt without necessarily retrieving its title typically reflects the existence of a memory system dedicated to the retrieval of musical knowledge. The functional distinction between musical and verbal semantic memory has seldom been investigated. In this fMRI study, we directly compared the musical and verbal memory of 20 nonmusicians, using a congruence task involving automatic semantic retrieval and a familiarity task requiring more thorough semantic retrieval. In the former, participants had to access their semantic store to retrieve musical or verbal representations of melodies or expressions they heard, in order to decide whether these were then given the right ending or not. In the latter, they had to judge the level of familiarity of musical excerpts and expressions. Both tasks revealed activation of the left inferior frontal and posterior middle temporal cortices, suggesting that executive and selection processes are common to both verbal and musical retrieval. Distinct patterns of activation were observed within the left temporal cortex, with musical material mainly activating the superior temporal gyrus and verbal material the middle and inferior gyri. This cortical organization of musical and verbal semantic representations could explain clinical dissociations featuring selective disturbances for musical or verbal material.
\end{abstract}

Keywords: inferior frontal cortex; fMRI; music; semantic memory; temporal cortex. 
Semantic memory refers to memory for general knowledge, unrelated to specific experiences or the type of material used (e.g. words, faces or music). Clinical studies have revealed that patients sometimes retain musical abilities despite severe cognitive impairments such as aphasia or amnesia (Signoret et al., 1987;Cuddy and Duffin 2005;Samson et al., 2009). Based on neuropsychological dissociations reported in clinical studies, Peretz and colleagues (Peretz and Coltheart 2003;Peretz et al., 2009) have developed a cognitive model of the cortical organization of music recognition. This views the musical semantic memory system as a purely musical lexicon, which interacts with the verbal lexicon. In this study, musical semantic memory is defined as the long-term storage of familiar melodies or musical excerpts. It is musical semantic memory that allows us to experience a strong feeling of knowing when listening to music (reflecting familiarity processes) and gives us the ability to hum or whistle the subsequent notes of a melody, or in some cases retrieve the title, composer or performer of a particular excerpt (corresponding to identification) (Platel and Eustache 2000). Whereas numerous clinical studies have supported the idea that musical knowledge and verbal knowledge are cognitively autonomous (for review, see Peretz 2008), few authors have investigated this issue using neuroimaging methods. The neural substrates of semantic memory have been unraveled using a variety of experimental paradigms in neuroimaging studies (Cabeza and Nyberg 2000; Binder et al., 2009). Semantic memory retrieval requires the activation of a large neural network, mainly located in the temporal and frontal cortices of the left hemisphere. When verbal material is used, semantic memory relies mainly upon the middle and inferior temporal and inferior frontal gyri in this hemisphere (for review, see Binder et al., 2009). The situation appears to be less clear-cut for musical material, however. Neuroimaging studies featuring this type of material have reported the involvement of the anterior part of the temporal lobes, either in the left hemisphere (Platel et al., 2003) or in both (Satoh et al. 2006), with activation of the middle part of the left superior temporal gyrus and the medial frontal cortices for recognition tasks (Satoh et al., 2006) and mainly of the left inferior frontal gyrus for familiarity tasks (Plailly et al., 2007). However, these studies did not allow direct comparisons to be made between music and language, and some of them used stimuli, such as nursery songs, which may also have elicited verbal processes.

In a previous $\mathrm{H}_{2} \mathrm{O}^{15}$ PET study, we found that the verbal and musical sets of material used in a semantic congruence task drew on two close but partially distinct networks, located mainly in 
the left temporal cortex (Groussard et al., 2010). In this study, participants had to decide whether the second part of a familiar melody (musical congruence condition) or a French proverb (verbal congruence condition) was the right or wrong ending. They therefore had to access their semantic store in order to retrieve musical or verbal representations of the melodies or expressions they heard in order to decide whether they were then given the right ending or not. The post-experiment debriefing suggested, however, that the congruence task involved syntactic processes as well (i.e. corresponding to the detection of irregularities within the harmonic, melodic, rhythmic or metric structure), particularly for incongruent items.

In order to clarify this issue and to highlight the brain regions mainly involved in the as pure semantic retrieval process as possible, we chose to administer the same semantic tasks in the present study, using fMRI. In fact, using an event-related analysis, this makes it possible to exclude incongruent items which could involve syntactic processes.

Regarding the literature, it is now well established that both the difficulty and nature of the semantic task have an impact on the pattern of activation (Mummery et al., 1996; Muller et al., 1997; Cabeza and Nyberg 2000). Thus, in order to take our direct comparison of musical and verbal semantic tasks a stage further, we decided to administer a "semantic familiarity task" as well, requiring a more thorough semantic memory search to rate the level of familiarity of musical excerpts (musical familiarity condition) and French expressions (verbal familiarity condition) on a 4-point scale. In all the musical conditions, familiar musical stimuli were strictly selected (no excerpts with lyrics, and no excerpts which might spontaneously evoke autobiographical memories) in order to limit labeling and verbalization. These two tasks were highly complementary, in that the congruence task allowed us to explore musical semantic processing with as few verbal associations as possible but was also limited to an automatic search, whereas the familiarity task gave rise to more thorough semantic retrieval, but opened up the possibility of verbal labeling when the participants knew the melody extremely well, corresponding to identification (retrieving the title, composer or performer of the musical excerpt). The complementary aspects of these two tasks (i.e. congruence and familiarity tasks) investigating automatic and more thorough semantic retrieval, performed by the same participants, would allow us to increase the understanding of the functional organization of musical semantic memory and highlight the neural networks activated by verbal material, musical material or both. Previous studies have shown that semantic memory tasks with verbal or musical material activated the prefrontal and temporal areas mainly on the left side for verbal (Binder et al., 2009) and in both hemispheres for musical material 
(Platel et al., 1997, 2003, Satoh et al., 2006 and Plailly et al., 2007). In addition, we recently proposed an anteroposterior organization within the left middle and superior temporal gyri (Groussard et al., 2010), such that there was predominantly anterior activation during the musical semantic task and predominantly posterior activation during the verbal one. Thus, comparing the performance of the same group of nonmusician participants on congruence and familiarity semantic tasks featuring verbal and musical material, allows to examin more deeply these networks and the cognitive contribution of each cortical area.

\section{MATERIAL \& METHODS}

\section{$\underline{\text { Participants }}$}

Twenty healthy right-handed volunteers (mean \pm SD: $24.55 \pm 3.80$ years) were selected from a population of university students (mean education level \pm SD: $16.35 \pm 2.03$ years) to take part in this study. All were nonmusicians (10 women and 10 men) with normal hearing and no history of neurological disease. Participants were selected according to stringent criteria: (1) none had taken music lessons or participated in musical performances (except for compulsory music classes at secondary school (1hr per week)), (2) they were "common listeners" (i.e. not music lovers, who tend to listen to one specific type of music only), and (3) they scored normally on a test of pitch perception. All gave their written informed consent prior to taking part and the research protocol was approved by the regional ethics committee.

\section{$\underline{\text { Stimuli and experimental procedure }}$}

All participants were tested on two memory tasks: a congruence task and a familiarity task. The order of these tasks was counterbalanced across participants.

\section{Congruence task}

In the congruence task, two similar categories of semantic memory tasks were performed: one using musical material (hereafter called "MusSem") and the other using verbal items ("VerbSem"). In the former, subjects heard the beginning of a well-known tune, followed by a short silence and a beep tone (mean interval $800 \mathrm{~ms}$ ), then either the next part of the melody or a different familiar melody. They had to determine whether the second part matched (i.e. was the right ending to) the first one or not. This musical semantic memory task was contrasted with a perceptual reference condition ("MusRef") in which the subjects listened to two unfamiliar sequences of notes that were either identical or differed by one note, and then had 
to say whether or not they were the same. This task was designed to call on the same decisional, perceptual and motor processes as the experimental task, but not on musical semantic memory, since the musical sequences were unknown to the participants.

In the verbal semantic memory task, subjects listened to the beginning of a French proverb or popular saying, followed by a short silence and a beep tone (mean interval $800 \mathrm{~ms}$ ), and then by either the right or wrong ending (one belonging to another proverb). They had to decide whether or not the second part matched the first one. This verbal semantic memory test was contrasted with a perceptual reference condition ("VerbRef") in order to subtract the brain activation produced by decisional, perceptual and motor processes. In this task, subjects had to indicate whether two meaningless sequences of syllables (nonwords respecting French phonological rules) were the same or if they differed by one syllable. The difficulty of the verbal and musical semantic tasks was tested and adjusted in a pilot study. The musical congruence task was designed to probe semantic processing with as few verbal associations as possible and thus, to make the contrast between melody and word processing as sharp as possible when comparing the activation results (no lyrics, and an instruction that did not involve trying to remember the title or composer of familiar melodies; see supplementary material). For each task, subjects were instructed to respond by pressing the button with their right index finger if the response was "correct" (musical and verbal semantic tasks) or "similar" (musical and verbal reference tasks) and with their right middle finger if it was "incorrect" (musical and verbal semantic tasks) or "different" (musical and verbal reference tasks).

The musical and verbal stimuli were presented in two functional runs of sixteen $31-34 \mathrm{~s}$ blocks. Each run consisted of four blocks in each condition (alternating between musical reference, musical semantic, verbal semantic and verbal reference). The response interval between two stimuli was set at 3 seconds in order to minimize automatic subvocal labeling or episodic memory processes during this time.

\section{Familiarity task}

Like the congruence task, the familiarity task featured both musical and verbal material. In this task, participants had to rate the level of familiarity of 60 excerpts of melodies and 60 French expressions on a 4-point scale (i.e. familiarity referring to the participants' life experience). Participants were instructed to press the button under the middle finger of their left hand if they were sure that they never had heard the melody or the expression before (Fam1), the button under the index finger of their left hand if they were not sure whether they 
had heard it before or not (Fam2), the button under the index finger of their right hand if they remembered hearing it on several occasions (Fam3) and the button under the middle finger of their right hand if they knew it extremely well (Fam4). Participants were instructed to close their eyes in order to focus more on the task. Melodies were purely instrumental tonal excerpts, taken from both the classical and modern repertoires. Popular songs and melodies associated with lyrics were avoided in order to minimize verbal associations, as were those which might spontaneously evoke autobiographical memories, such as the "Wedding March" or melodies used in popular TV commercials. All melodies were selected from a previous pilot study whose participants were matched with the present task subject sample. The musical and verbal stimuli used in this familiarity task were different from those used in the congruence task (see supplementary material). The familiarity processes involved in this task required recognition not just of the item's perceptual features, but also of its conceptual or semantic features. After a short training session performed inside the scanner, participants underwent two runs, each lasting 4 minutes and including 60 stimuli (30 verbal and 30 musical). The order of presentation of the conditions (Verbal, Music) was determined according to an efficient stochastic design (Friston et al., 1999) and the optimum order for detecting differences between the verbal and music items was computed using a genetic algorithm (Wager and Nichols, 2003).

All the melodies were played on a digital keyboard with a flute voice without orchestration and lasted 5-6 s. Before each fMRI scanning session (i.e. congruence task plus familiarity task), the subjects were trained with stimuli that were not used during the scanning session. To allow direct comparisons to be made, the verbal and musical excerpts belonging to the same experiment were delivered to each participant in the same scanning session.

The musical stimuli were delivered through electrodynamic headphones ensuring attenuation of scanner noise by as much as 45dB (MR Confon headphones, Magdeburg, Germany). The volume of the musical stimuli was adjusted to ensure that each subject could hear the stimuli clearly above the noise of the MRI scanner. Melodies were produced using E-Prime software (Psychology Software Tools, Pittsburgh, PA) implemented within IFIS (Invivo, Orlando, FL). Following the scanning session, we performed a debriefing in order to determine whether the melodies and expressions evoked any personal memories or mental imagery for the participants. 


\section{$\underline{\text { Behavioral analyses }}$}

\section{Congruence task}

Using Statistica software, we first carried out a repeated-measures analysis of variance (ANOVA) on performances. A two-way ANOVA was then conducted, with material as a between-subjects factor with two modalities (verbal and musical), and type of task as a within-subjects factor with two modalities (reference and semantic). If a significant interaction effect was observed, these analyses were followed up with Tukey's HSD post hoc tests.

\section{$\underline{\text { Familiarity task }}$}

Once again, a two-way ANOVA was performed, with material as a between-subjects factor with two modalities (verbal and musical), and familiarity as a within-subjects factor with four modalities (Fam1, Fam2, Fam3 and Fam4). Similar analyses were conducted with response time as a within-subjects factor with the same four modalities as those described above. If a significant interaction effect was observed, these analyses were followed up with Tukey's HSD post hoc tests.

\section{$\underline{\text { MRI scans }}$}

All images were acquired using the Philips (Eindhoven, The Netherlands) Achieva 3.0 T scanner. For each participant, a high-resolution, T1-weighted anatomical image was first acquired using a 3D fast field echo sequence (3D-T1-FFE sagittal, $\mathrm{TR}=20 \mathrm{~ms}$; $\mathrm{TE}=4.6 \mathrm{~ms}$; flip angle $=20^{\circ} ; 170$ slices; slice thickness $=1 \mathrm{~mm} ; \mathrm{FOV}=256 \times 256 \mathrm{~mm}^{2} ;$ matrix $=256 \mathrm{x}$ 256; acquisition voxel size $=1 \times 1 \times 1 \mathrm{~mm}^{3}$ ), followed by a high-resolution, T2-weighted anatomical image (2D-T2-SE sagittal, SENSE factor $=2$; TR $=5500 \mathrm{~ms}$; TE $=80 \mathrm{~ms}$; flip angle $=90^{\circ} ; 81$ slices; slice thickness $=2 \mathrm{~mm} ; \mathrm{FOV}=256 \times 256 \mathrm{~mm}^{2} ;$ matrix $=256 \times 256$; acquisition voxel size $=2 \times 1 \times 1 \mathrm{~mm}^{3}$ ) and a non-echo-planar (non-EPI) T2* image (2D-T2* FFE axial, SENSE factor $=2 ; \mathrm{TR}=3505 \mathrm{~ms} ; \mathrm{TE}=30 \mathrm{~ms}$; flip angle $=90^{\circ} ; 70$ slices; slice thickness $=2 \mathrm{~mm} ; \mathrm{FOV}=256 \times 256 \mathrm{~mm}^{2} ;$ matrix $=128 \times 128$; acquisition voxel size $=2 \times 2$ $\left.\mathrm{x} 2 \mathrm{~mm}^{3}\right)$.

Functional data were acquired using an interleaved 2D T2* EPI sequence designed to reduce geometric distortion and magnetic susceptibility artefacts (2D-T2*-FFE-EPI axial, SENSE factor $=2$; $\mathrm{TR}=2382 \mathrm{~ms} ; \mathrm{TE}=30 \mathrm{~ms}$; flip angle $=80^{\circ} ; 44$ slices; slice thickness $=$ $2.8 \mathrm{~mm}$; matrix $=80 \times 80 ; \mathrm{FOV}=224 \times 224 \mathrm{~mm}^{2}$; acquisition voxel size $=2.8 \times 2.8 \times 2.8$ $\mathrm{mm}^{3} ; 216$ volumes per run for the congruence task and 207 for familiarity). The functional 
volumes were collected during two functional sessions for each experiment (i.e. congruence and familiarity tasks). The six initial scans of each session were discarded to control for magnetic effects.

\section{fMRI data processing}

Data were analyzed using statistical parametric mapping software (SPM5; Wellcome Department of Cognitive Neurology, Institute of Neurology, London, UK). In the preprocessing steps, images were corrected for slice timing and then realigned on the first volume of the first run. Subsequently, coregistration of the EPI volumes onto the T1 image was done in 3 steps: (1) the non-EPI T2* volume was first coregistered to the mean EPI image for the two runs, (2) the T2 image was then coregistered to the coregistered non-EPI T2* volume, and finally (3) the T1 volume was coregistered to the coregistered T2 image. Images were warped to roughly match the non-EPI T2* volume using the methodology developed and validated by Villain et al. (2010) to reduce geometric distortion. The warping parameters were then applied to all the EPI volumes for the session. The T1 image was then segmented/normalized using the SPM5 'Segment' procedure (Ashburner and Friston 2005), with the ICBM/MNI priors and the resulting normalization parameters were applied to the T1 to the T2 Star normalized EPI images and to the non-EPI T2 Star volume. The normalized EPI images were finally smoothed at $8 \mathrm{~mm}$ FWHM. A high-pass filter was implemented using a cut-off period of $128 \mathrm{~s}$ to remove low-frequency drift from the time series.

Data were then analyzed using the general linear model approach on a voxel-by-voxel basis with a random effects model implemented with a two-level procedure, for both the congruence and the familiarity experiments. The aim of our study was understand the functional organization of semantic memory more fully, particularly the distinction between the semantic retrieval of musical and verbal material. For this purpose we chose to restrict our investigations to contrasts that revealed semantic retrieval processes and allowed the direct comparison of musical and verbal material.

\section{fMRI data analysis}

For each participant, changes in condition-related BOLD responses were estimated first, in the intra-individual analysis. For each experimental condition, the hemodynamic response was estimated by convolving the stimuli onset with the canonical hemodynamic response function (HRF). Motion parameters obtained from the realignment procedure were included as covariates of no interest in the design matrix. 


\section{Congruence task}

Several contrasts (e.g. MusSem-MusRef; VerbSem-VerbRef; MusFam-VerFam; VerbFam-MusFam) were computed for each subject. In these contrasts, only the congruent stimuli were included, in order to reduce possible contamination by incongruity detection processes. All resulting contrast images were then entered into second-level random effect analyses.

For the congruence experiment, a full factorial design was used to reveal brain activation specifically associated with musical and verbal semantic memory processes. We performed the direct comparison between the two semantic tasks after subtracting the respective reference tasks [MusSem-MusRef] - [VerbSem-VerbRef] (masked exclusively by the [VerbRef-VerbSem] contrast), to highlight brain activation specifically associated with musical semantic memory processes, excluding both the effects of perceptual activity and contamination by verbal semantic components. We also performed the reverse comparison [VerbSem-VerbRef] - [MusSem-MusRef] (masked exclusively by the MusRef $>$ MusSem] contrast) which we expected to highlight the neural substrates of verbal semantic memory.

To identify the brain areas involved in both the verbal semantic task versus verbal reference task [VerbSem-VerbRef] and the musical semantic task versus musical reference task [MusSem-MusRef] comparisons, we performed a conjunction analysis. This analysis served to pinpoint the cerebral network common to both musical and verbal semantic processes. We used a conjunction analysis based on the "valid conjunction inference with the minimum statistic" procedure (Nichols et al., 2005). Each comparison in the conjunction was individually significant, corresponding to the valid test for a "logical AND".

\section{Familiarity task}

Two contrasts (e.g. Musical-Verbal and Verbal-Musical) were computed for each subject, and all resulting contrast images were then entered into second-level random effect analyses using a one-sample $t$-test to highlight differences between the verbal and musical semantic networks.

To investigate the effects of familiarity on the parametric modulation of the canonical hemodynamic response function, a parametric regressor modeling familiarity was constructed on the basis of the familiarity judgments for each song excerpt and each French expression in the intra-individual level analysis. Familiarity was rated on a 4-point scale (from unfamiliar (1) to very familiar (4)). This analysis, performed for each subject, allowed us to identify 
brain areas where activation increased with familiarity for each type of material (MusicFam and VerbalFam). An explicit mask was used to exclude activation due to a motor response. Then, in a second-level, group analysis, a random effects analysis was conducted using a one sample $t$-test.

The resulting set of voxel values was threshold at $p<0.001$ (uncorrected) and we only report activation involving clusters of voxels above 50. This statistical threshold was chosen in the light of empirical studies showing that this threshold protects against false positives (Bailey et al., 1991).

\section{RESULTS}

\section{Behavioral data}

\section{Congruence task}

Mean accuracy of performance was $83.71 \%(\mathrm{SD}=8.72$ ) for the musical semantic task, $98.71 \%(\mathrm{SD}=2.85)$ for the verbal semantic task, and $94.06 \%(\mathrm{SD}=5.26)$ and $93.42 \%(\mathrm{SD}=$ 3.94) for the musical and verbal reference tasks. Accuracy was significantly lower for the musical semantic task than for either the verbal semantic task or either of the reference tasks $(p<0.001)$. These performances were not significantly different from those of the subjects in our pre-experimental population.

\section{Familiarity task}

The statistical analysis (ANOVA) performed on familiarity ratings revealed (1) no main effect of material, $F(1,38)=1.24, p=0.27$, (2) a main effect of familiarity, $F(3,114)=38.23, p<$ 0.001 , and (3) a significant effect of the interaction between material and familiarity, $F(3$, $114)=34.95, p<0.001$. On the basis of this finding, post hoc comparisons (Tukey's HSD) were performed in order to identify specific effects. We observed a significant difference $(p<$ 0.001) in the number of verbal and musical stimuli rated as Fam2, Fam3 and Fam4. As might have been expected with nonmusicians, participants found more verbal than musical samples to be "very familiar" (Fam 4).

A similar analysis of response times revealed (1) a main effect of material, $F(1,38)=165.59$, $p<0.001$, with longer response times for music than for words, (2) a main effect of familiarity, $F(3,114)=94.50, p<0.001$, with longer response times when subjects were not sure whether they had heard the item before or not (i.e. Fam 2) and (3) an effect of the interaction between the material and familiarity factors, $F(3,114)=10.69, p<0.001$. To sum 
up, the more familiar the stimuli, the shorter the response times for both musical and verbal material.

\section{fMRI data}

\section{Congruence task}

The musical semantic contrast ([SemMus-RefMus]-[SemVerb-RefVerb], Figure 1, red, Table1) bilaterally activated the superior temporal gyrus (BA 22), extending to the superior part of the middle temporal gyrus, the inferior frontal gyrus (BA 44) (i.e. pars triangularis and orbital part), the inferior part of the parietal lobule (BA 40/39), and the supplementary motor area (upper part of BA 6). On the left side, we also obtained activation of the insula (BA 47) and cerebellum.

The verbal semantic contrast ([SemVerb-RefVerb]-[SemMus-RefMus], Figure 1, blue, Table 1) revealed only left-sided activation, in the angular gyrus (BA 39) (extending to the middle portion of the temporal gyrus), the anterior half of the middle and inferior temporal gyri (BA 21 ), and the anterior part of the medial frontal cortex, including parts of the orbitofrontal (BA 9/10) and cingulate gyri.

The conjunction analysis (Figure 1, yellow, Table 1) revealed left-sided activation, common to both the verbal and the musical tasks, in the posterior middle temporal area (BA21), the posterior inferior frontal gyrus (BA45/47, including Broca's area) and, to a lesser extent, the middle frontal gyrus (BA6), and the right cerebellum.

\section{Familiarity task}

The musical contrast ([Mus-Verb], Figure 2, red, Table 2) revealed bilateral activation in the superior temporal gyri (BA 22) (extending into Heschl's gyri), left middle cingulate and right insula (BA 47).

The verbal contrast ([Verb-Mus], Figure 2, blue, Table 2) highlighted mostly left-sided activation, in the inferior and middle temporal gyri (BA 21) (extending to the inferior part of the inferior orbitofrontal gyrus), the medial superior (BA 10) and inferior pars triangularis frontal gyri (BA 45), the posterior middle temporal gyrus and, on the right side, the inferior orbitofrontal cortex (BA 47), the middle temporal pole (BA 38), the anterior part of the hippocampus and the cerebellum. 
Effect of familiarity on the parametric modulation of the Hemodynamic Response Function $\underline{(H R F)}$

To highlight the effect of familiarity, a parametric regressor modeling familiarity was constructed, in order to highlight those areas where activity increased with familiarity. This analysis revealed greater activity for music (Figure 3, Table 3) in an extended network that included the bilateral inferior frontal gyrus (BA45) (extending to the middle frontal gyrus), the posterior part of the inferior and middle temporal gyri (BA20/37), the medial superior frontal gyrus (BA10) and the right superior temporal pole (BA38). The verbal familiarity analysis revealed greater activity in the left medial superior frontal gyrus (BA10), the middle cingulate cortex bilaterally, the left putamen and thalamus, and the inferior and middle parts of the frontal (BA47/46) and temporal gyri (BA20/21) bilaterally. We also found activation of the posterior part of the right hippocampus and the left parahippocampal cortex.

\section{DISCUSSION}

The possibility of there being a neural distinction between verbal and musical material has been raised by several clinical studies (e.g. Signoret et al., 1987; Eustache et al., 1990; Peretz 2002) but has seldom been investigated in neuroimaging studies. Direct comparisons between language and music have rarely been performed so far and focused mainly on perceptual, production and syntactic processing (Patel 2003; Koelsch et al., 2005; Brown et al., 2006; Ozdemir et al., 2006; Steinbeis and Koelsch 2008). According to these studies, a number of brain areas, among which the left prefrontal cortex (BA44/45, Koelsch et al., 2004;Maess et al., 2001), appear to be involved in both language and music particularly in the syntactic processing. So far, until now, few neuroimaging studies have supported the existence of an independent musical network, corresponding to the musical lexicon postulated by Peretz and collaborators on the basis of neuropsychological observations (Peretz and Coltheart 2003). The results of our recent PET activation study suggest that such a distinction does indeed exist for verbal and musical semantic memory processes (Groussard et al., 2010). Using two semantic tasks (congruence and familiarity), we now confirm our previous results and increase the knowledge of the functional organization of the processes involved in musical semantic retrieval. In fact, using fMRI, we revealed partially segregated neural networks for musical and verbal semantic memory retrieval within the frontal and temporal gyri in the left 
hemisphere, whichever semantic task was used. We first address the neural substrates underlying musical semantic memory and their specific functions. Then, we discuss the cerebral areas shared by the musical and verbal processes and the specific ones.

\section{Musical semantic memory network}

Consistent with previous neuroimaging studies using musical semantic memory tasks (Satoh et al., 2006; Plailly et al., 2007; Groussard et al., 2010), we observed bilateral activation for musical material, mainly in temporal and frontal areas. The complementary nature of the tasks used in this study, allowed to further highlight the various processes involved in musical semantic retrieval and their neural substrates.

The congruence task, which required participants to decide whether the second part matched (i.e. was the right ending for) the first part or not, revealed ([SemMus-RefMus]-[SemVerbRefVerb], Figure 1, Table 1) a network encompassing the bilateral inferior frontal (BA 44/45) and superior temporal gyri (BA22), with additional activation in the inferior part of the parietal gyrus bilaterally (BA40) and the left insula (BA47). The familiarity task, which required participants to compare the melodies they heard with those already stored in their semantic memory, revealed adjacent activation in the superior temporal gyri (BA48) bilaterally (Mus-Verb; Figure 2, Table 2) and in the inferior frontal (BA45/47) and medial superior frontal gyri (BA10) on both sides (musical familiarity analysis; Figure 3, Table 3).

The activation of the left frontal cortex has already been highlighted using different types of musical material and experimental paradigms, including a musical recognition task (Satoh et al., 2006; Watanabe et al., 2008), a musical familiarity task (Platel et al., 1997; Platel et al., 2003; Plailly et al., 2007) and a musical syntactic task (Tillmann et al., 2003; Koelsch et al., 2005). In the light of all these findings, the left inferior frontal areas (BA44/45/47) would appear to play a crucial role in musical retrieval processes, possibly reflecting the selection and recapitulation processes recruited to solve the task. While retrieving memory for musical material, the participants had to retain the melody they had heard in order to compare it with those already stored in their semantic memory, and inhibit all irrelevant representations (Badre and Wagner 2007). These are the processes that were predominantly involved in our congruence and familiarity experiments, consistent with the extended activation observed in the left inferior frontal area (Figure 1 and 3). In addition, the left inferior frontal activation observed when familiarity increased (Figure 3) could reflect successful recognition in semantic memory, particularly for the anterior part (BA 45) of this area (Watanabe et al., 2008; Hayama and Rugg 2009). The right inferior frontal activation observed in both 
experiments may also have reflected semantic processes, but were probably associated with imagery and internal decision-making processes. The right frontal cortex is known to reflect the musical imagery evoked on hearing a familiar melody (Halpern and Zatorre 1999) and the internal decisions that precede response selection (Hayama and Rugg 2009). Although we selected stimuli with as few emotional associations as possible, listening to musical excerpts inevitably induced various emotional reactions. Our data also revealed activation in areas classically involved in emotion, particularly the insula and the cingulate cortex (Blood and Zatorre 2001; Brown et al., 2004; Stewart et al., 2006).

The activation of the superior temporal gyri (BA 22) obtained in the musical congruence and musical familiarity tasks (Figure 1 and 2) extends our previous results (Platel et al., 1997; Platel et al., 2003; Groussard et al., 2010) and is consistent with previous musical memory studies (Halpern and Zatorre 1999; Satoh et al., 2006; Watanabe et al., 2008). These areas play a crucial role in musical semantic memory, whatever semantic task is used. The right superior temporal gyrus was mainly highlighted by the familiarity analysis (Music vs. Verbal without subtracting perceptual processes) and may reflect the musical perceptual processes that precede semantic retrieval (see Zatorre 2003). Chen et al., (2008) reported posterior temporal gyrus activation in the right hemisphere during the perception of and synchronization with musical rhythms, and interpreted it as meaning that this area is a "computational hub". Given that information about rhythm, as well as pitch, serves as the basis for deciding whether or not a melody is familiar (Kostic and Cleary 2009), and given the results of our previous study (Groussard et al., 2010), we hypothesize that the right temporal cortex, especially its superior part, is mainly involved in the retrieval of perceptual memory traces.

The left superior temporal gyrus was flagged up in all our previous musical memory studies (Platel et al., 1997; Platel et al., 2003;Groussard et al., 2010), as well as in the present congruence and familiarity experiments. This area seems to be linked to access to nonverbal semantic attributes and knowledge of familiar tunes, processes involved in distinguishing between familiar and unfamiliar melodies. Dalla Bella et al., (2003) suggested breaking the music recognition process down into two stages: access and selection. In the light of our results, we suggest that the left superior temporal gyrus subserves the access stage and the inferior frontal area the selection one. 


\section{Musical and verbal memory: two neural networks?}

The possibility of there being a neural distinction between music and language was initially suggested by clinical dissociations (for review, see Peretz 2008), but has rarely been investigated in neuroimaging studies using comparable semantic memory tasks featuring musical and verbal material. Our previous study (Groussard et al., 2010) revealed a neural dissociation between language and music for semantic memory processes. In the present study, we used two complementary semantic tasks to confirm this finding and to understand more fully the functional organization of the various processes involved in semantic retrieval, particularly for musical material.

In the congruence task, the conjunction analysis revealed regions involved in both musical and verbal processing, such as the left frontal inferior area, which is activated by syntactic and semantic tasks featuring both verbal and musical material (Platel et al., 2003; Koelsch 2006; Steinbeis and Koelsch 2008; Binder et al., 2009;Groussard et al., 2010). Consistent with Brown et al.'s proposition (2006), we suggest that the left inferior frontal area (BA 44/45) subserves amodal executive and recapitulation processes involved in performing semantic memory tasks whichever type of material is used. Badre and Wagner (2007) interpreted the role of the inferior frontal cortex as subserving the top-down control of semantic information to perform a memory task. Regarding the existence of interactions between the inferior frontal cortex and the posterior part of the middle temporal lobe during conceptual recognition (Kuhl and Wagner 2009), it is not surprising that activation of the left posterior middle temporal cortex also appeared to be common to music and language in our semantic tasks. Our results confirm those reported by Brown et al., (2006) when they compared music and language in a melody and sentence generation task. These authors found that the posterior part of the left middle temporal gyrus was activated for both language and music, and concluded that this area serves as a key phonological/semantic interface, in verbal semantic processing.

Using two semantic memory tasks in an fMRI investigation, we found that musical semantic memory and verbal semantic memory are subserved by two distinct networks. As explained below, our present study suggested that the musical retrieval network mainly encompasses the bilateral superior temporal areas, while the verbal retrieval network ([SemVerb-RefVerb][SemMus-RefMus] and [Verb-Mus], Figure 1, Table 1) comprises the left inferior and middle temporal gyri. These findings are consistent with activation patterns revealed in verbal semantic memory studies (for review, see Cabeza and Nyberg 2000; Binder et al., 2009). Our results support Brown et al.'s proposal (2006) that the left superior temporal gyrus is a plausible candidate for representing the semantics of music. Moreover, the verbal familiarity 
task revealed specific, bilateral activation of the hippocampal areas. These results are consistent with the involvement of the hippocampal structures in familiarity processes postulated by a number of authors (Squire et al., 2004; Daselaar et al., 2006; Diana et al., 2007; Svoboda and Levine 2009; Burianova et al., 2010).

In our previous study (Groussard et al., 2010), we suggested that verbal and musical types of material rely on two different networks and proposed an anteroposterior organization within the temporal cortex for semantic concepts, with musical semantic retrieval involving the anterior temporal lobe more than its verbal equivalent. Using a more sensitive neuroimaging technique and two semantic experiments performed by the same population, we were able to confirm the existence of a neural distinction between language and music material during semantic memory retrieval. Overall, our present data suggest that the temporal cortex is organized along an inferior/superior axis depending of the nature of the material being retrieved. The musical material mainly activated the superior temporal cortex, whereas the middle and inferior temporal cortex was activated by the verbal material. Moreover, the anteroposterior organization suggested by our previous study (Groussard et al., 2010) could be added to the inferior/superior organization. This anteroposterior distribution appears to be dependent on the nature (unique or general) of the semantic representation to retrieve. Thus, the representations of musical material could be considered as unique semantically unique (such as faces or famous buildings) because of their personal specificity, whereas verbal material refers to more general semantic representations shared by everybody and associated with several concepts.

In addition, this study allowed us to show that a number of areas are activated by both verbal and musical semantic memory processes. Verbal and musical semantic retrieval share the same executive and selection processes, which are subserved by the left inferior frontal cortex. This inferior/superior organization within the left temporal cortex could help understanding the clinical dissociations that are observed (Piccirilli et al., 2000) and the disproportionate preservation of musical knowledge in semantic dementia contrasting with severely impaired verbal skills (Hailstone et al., 2009).

Acknowledgments: This study was supported by a "Music and Memory" French National Research Agency (ANR) grant (NT05-3_45987) and by the French Ministry of Research. We thank N. Villain, P. Gagnepain and G. Chételat for their valuable contribution, and C. 
Mauger, J. Dayan, C. Schupp and the neuroimaging staff of the Cyceron center for their help with data acquisition.

\section{REFERENCES}

Ashburner, J., Friston, K.J., 2005. Unified segmentation. Neuroimage. 26, 839-851.

Badre, D., Wagner, A.D., 2007. Left ventrolateral prefrontal cortex and the cognitive control of memory. Neuropsychologia. 45, 2883-2901.

Bailey, D.L., Jones, T., Spinks, T.J., 1991. A method for measuring the absolute sensitivity of positron emission tomographic scanners. Eur J Nucl Med. 18, 374-379.

Binder, J.R., Desai, R.H., Graves, W.W., Conant, L.L., 2009. Where is the semantic system? A critical review and meta-analysis of 120 functional neuroimaging studies. Cereb Cortex. 19, 2767-2796.

Blood, A.J., Zatorre, R.J., 2001. Intensely pleasurable responses to music correlate with activity in brain regions implicated in reward and emotion. Proc Natl Acad Sci U S A. 98, 11818-11823.

Brown, S., Martinez, M.J., Parsons, L.M., 2004. Passive music listening spontaneously engages limbic and paralimbic systems. Neuroreport. 15, 2033-2037.

Brown, S., Martinez, M.J., Parsons, L.M., 2006. Music and language side by side in the brain: a PET study of the generation of melodies and sentences. Eur J Neurosci. 23, 27912803.

Burianova, H., McIntosh, A.R., Grady, C.L., 2010. A common functional brain network for autobiographical, episodic, and semantic memory retrieval. Neuroimage. 49, 865-874.

Cabeza, R., Nyberg, L., 2000. Imaging cognition II: an empirical review of 275 PET and fMRI studies. J Cogn Neurosci. 12, 1-47.

Chen, J.L., Penhune, V.B., Zatorre, R.J., 2008. Listening to musical rhythms recruits motor regions of the brain. Cereb Cortex. 18, 2844-2854.

Cuddy, L.L., Duffin, J., 2005. Music, memory, and Alzheimer's disease: is music recognition spared in dementia, and how can it be assessed? Med Hypotheses. 64, 229-235. 
Dalla Bella, S., Peretz, I., Aronoff, N., 2003. Time course of melody recognition: a gating paradigm study. Percept Psychophys. 65, 1019-1028.

Daselaar, S.M., Fleck, M.S., Cabeza, R., 2006. Triple dissociation in the medial temporal lobes: recollection, familiarity, and novelty. J Neurophysiol. 96, 1902-1911.

Diana, R.A., Yonelinas, A.P., Ranganath, C., 2007. Imaging recollection and familiarity in the medial temporal lobe: a three-component model. Trends Cogn Sci. 11, 379-386.

Eustache, F., Lechevalier, B., Viader, F., Lambert, J., 1990. Identification and discrimination disorders in auditory perception: a report on two cases. Neuropsychologia. 28, 257270.

Friston, K.J., Zarahn, E., Josephs, O., Henson, R.N., Dale, A.M., 1999. Stochastic designs in event-related fMRI. Neuroimage. 10, 607-619.

Groussard, M., Viader, F., Hubert, V., Landeau, B., Abbas, A., Desgranges, B., Eustache, F., Platel, H., 2010. Musical and verbal semantic memory: Two distinct neural networks? Neuroimage. 49, 2764-2773.

Hailstone, J.C., Omar, R., Warren, J.D., 2009. Relatively preserved knowledge of music in semantic dementia. J Neurol Neurosurg Psychiatry. 80, 808-809.

Halpern, A.R., Zatorre, R.J., 1999. When that tune runs through your head: a PET investigation of auditory imagery for familiar melodies. Cereb Cortex. 9, 697-704.

Hayama, H.R., Rugg, M.D., 2009. Right dorsolateral prefrontal cortex is engaged during postretrieval processing of both episodic and semantic information. Neuropsychologia. 47, 2409-2416.

Koelsch, S., Kasper, E., Sammler, D., Schulze, K., Gunter, T., Friederici, A.D., 2004. Music, language and meaning: brain signatures of semantic processing. Nat Neurosci. 7, 302307.

Koelsch, S., Gunter, T.C., Wittfoth, M., Sammler, D., 2005. Interaction between syntax processing in language and in music: an ERP Study. J Cogn Neurosci. 17, 1565-1577.

Koelsch, S., 2006. Significance of Broca's area and ventral premotor cortex for musicsyntactic processing. Cortex. $42,518-520$. 
Kostic, B., Cleary, A.M., 2009. Song recognition without identification: when people cannot "name that tune" but can recognize it as familiar. J Exp Psychol Gen. 138, 146-159.

Kuhl, B.A., Wagner, A.D., 2009. Strategic control of memory. In: Squire LR, editor. Encyclopedia of Neuroscience. Oxford (England): Academic Press, p 437-444.

Maess, B., Koelsch, S., Gunter, T.C., Friederici, A.D., 2001. Musical syntax is processed in Broca's area: an MEG study. Nat Neurosci. 4, 540-545.

Muller, R.A., Rothermel, R.D., Behen, M.E., Muzik, O., Mangner, T.J., Chugani, H.T., 1997. Receptive and expressive language activations for sentences: a PET study. Neuroreport. 8, 3767-3770.

Mummery, C.J., Patterson, K., Hodges, J.R., Wise, R.J., 1996. Generating 'tiger' as an animal name or a word beginning with T: differences in brain activation. Proc Biol Sci. 263, 989-995.

Nichols, T., Brett, M., Andersson, J., Wager, T., Poline, J.B., 2005. Valid conjunction inference with the minimum statistic. Neuroimage. 25, 653-660.

Ozdemir, E., Norton, A., Schlaug, G., 2006. Shared and distinct neural correlates of singing and speaking. Neuroimage. 33, 628-635.

Patel, A.D., 2003. Language, music, syntax and the brain. Nat Neurosci. 6, 674-681.

Peretz, I., 2002. Brain specialization for music. Neuroscientist. 8, 372-380.

Peretz, I., Coltheart, M., 2003. Modularity of music processing. Nat Neurosci. 6, 688-691.

Peretz, I., 2008. Music, language and modularity in action. P. Rebuschat, M. Rohrmeier, J.Hawkins, and I.Cross (Eds.), Language and music as cognitive systems. Oxford University Press.

Peretz, I., Gosselin, N., Belin, P., Zatorre, R.J., Plailly, J., Tillmann, B., 2009. Music lexical networks: the cortical organization of music recognition. Ann N Y Acad Sci. 1169, 256-265.

Piccirilli, M., Sciarma, T., Luzzi, S., 2000. Modularity of music: evidence from a case of pure amusia. J Neurol Neurosurg Psychiatry. 69, 541-545. 
Plailly, J., Tillmann, B., Royet, J.P., 2007. The feeling of familiarity of music and odors: the same neural signature? Cereb Cortex. 17, 2650-2658.

Platel, H., Price, C., Baron, J.C., Wise, R., Lambert, J., Frackowiak, R.S., Lechevalier, B., Eustache, F., 1997. The structural components of music perception. A functional anatomical study. Brain. 120, 229-243.

Platel, H., Baron, J.C., Desgranges, B., Bernard, F., Eustache, F., 2003. Semantic and episodic memory of music are subserved by distinct neural networks. Neuroimage. 20, 244256.

Platel, H., 2005. Functional neuroimaging of semantic and episodic musical memory. Ann N Y Acad Sci. 1060, 136-147.

Samson, S., Dellacherie, D., Platel, H., 2009. Emotional power of music in patients with memory disorders: clinical implications of cognitive neuroscience. Ann N Y Acad Sci. $1169,245-255$.

Satoh, M., Takeda, K., Nagata, K., Shimosegawa, E., Kuzuhara, S., 2006. Positron-emission tomography of brain regions activated by recognition of familiar music. AJNR Am J Neuroradiol. 27, 1101-1106.

Signoret, J.L., van Eeckhout, P., Poncet, M., Castaigne, P., 1987. [Aphasia without amusia in a blind organist. Verbal alexia-agraphia without musical alexia-agraphia in Braille]. Rev Neurol (Paris). 143, 172-181.

Squire, L.R., Stark, C.E., Clark, R.E., 2004. The medial temporal lobe. Annu Rev Neurosci. 27, 279-306.

Steinbeis, N., Koelsch, S., 2008. Comparing the processing of music and language meaning using EEG and fMRI provides evidence for similar and distinct neural representations. PLoS ONE. 3:e2226.

Stewart, L., von Kriegstein, K., Warren, J.D., Griffiths, T.D., 2006. Music and the brain: disorders of musical listening. Brain. 129, 2533-2553.

Svoboda, E., Levine, B., 2009. The effects of rehearsal on the functional neuroanatomy of episodic autobiographical and semantic remembering: a functional magnetic resonance imaging study. J Neurosci. 29, 3073-3082.

Tillmann, B., Janata, P., Bharucha, J.J., 2003. Activation of the inferior frontal cortex in musical priming. Ann N Y Acad Sci. 999, 209-211. 
Villain, V., Landeau, B., Groussard, M., Mevel, K., Fouquet, M., Dayan, J., Eustache, F., Desgranges, B., Chételat, G., 2010. A simple way to improve anatomical mapping of functional brain imaging. J Neuroimaging.

Wager, T.D., Nichols, T.E., 2003. Optimization of experimental design in fMRI: a general framework using a genetic algorithm. Neuroimage. 18, 293-309.

Watanabe, T., Yagishita, S., Kikyo, H., 2008. Memory of music: roles of right hippocampus and left inferior frontal gyrus. Neuroimage. 39, 483-491.

Zatorre, R.J., 2003. Music and the brain. Ann N Y Acad Sci. 999, 4-14. 


\begin{tabular}{|llllll|}
\hline Contrast and anatomical location & $\begin{array}{l}\text { Cluster size } \\
\text { (in voxels) }\end{array}$ & $\mathbf{x}$ & $\mathbf{y}$ & $\mathbf{z}$ & Z score \\
\hline [MusSem-MusRef]-[VerbSem-VerbRef] & & & & & \\
Left superior and middle temporal (BA22) & 201 & -58 & -28 & 4 & 5.86 \\
Right inferior frontal (BA44/45) & 1431 & 58 & 20 & 24 & 5.51 \\
Left inferior frontal/insula (BA47) & 413 & -36 & 26 & -4 & 5.20 \\
Right superior and middle temporal (BA22) & 342 & 48 & -26 & -6 & 4.93 \\
Left inferior frontal (BA48/44) & 763 & -52 & 16 & 24 & 4.93 \\
Right inferior parietal/angular (BA39/40) & 503 & 40 & -58 & 48 & 4.31 \\
Left inferior parietal (BA40) & 143 & -46 & -46 & 48 & 4.09 \\
Supplementary motor area (BA6) & 159 & 2 & 12 & 62 & 3.98 \\
Left cerebellum crus1 & 89 & -34 & -68 & -24 & 3.95 \\
\hline IVerbSem-VerbRef]-[MusSem-MusRef] & & & & & \\
Left angular (BA39) & 478 & -54 & -62 & 28 & 5.07 \\
Left superior frontal (BA9) & 498 & -12 & 52 & 38 & 4.79 \\
Left middle temporal (BA21) & 339 & -56 & -4 & -20 & 4.77 \\
Left middle cingulate & 54 & -16 & -46 & 32 & 4.22 \\
Left medial superior frontral (BA10) & 100 & -8 & 58 & 4 & 4.09 \\
\hline Conjunction (MusSem-MusRef) and (VerbSem-Verb Ref) & & & & \\
Left middle temporal (BA21) & 379 & -56 & -38 & -4 & 5.87 \\
Left middle frontal (BA6) & 252 & -28 & 2 & 50 & 4.87 \\
Left inferior frontal (BA45/47) & 490 & -46 & 28 & -8 & 4.60 \\
Right cerebellum crus1,2 & 81 & 12 & -82 & -28 & 4.35 \\
\hline
\end{tabular}

Table 1: Brain regions activated during the congruence experiment. $\mathrm{x}, \mathrm{y}, \mathrm{z}$ coordinates $(\mathrm{mm})$ are given in standard stereotactic MNI space. All regions listed are statistically at the $p<$ 0.001 level (uncorrected).

\begin{tabular}{|llllll|}
\hline Contrast and anatomical location & $\begin{array}{l}\text { Cluster size } \\
\text { (in voxels) }\end{array}$ & $\mathbf{x}$ & $\mathbf{y}$ & $\mathbf{Z}$ & Z score \\
\hline Music-Verbal & & & & & \\
Right superior temporal (BA22) & 1569 & 52 & -10 & 2 & 7.06 \\
Left superior temporal (BA22) & 1876 & -46 & -16 & 4 & 6.99 \\
Right insula (BA47) & 60 & 36 & 32 & 0 & 4.32 \\
Left middle cingulate & 64 & -2 & -20 & 30 & 4.05 \\
\hline Verbal-Music & & & & & \\
Left middle temporal (BA21) & 4802 & -52 & 4 & -20 & 6.10 \\
Right inferior frontal (BA47) & 185 & 50 & 42 & -14 & 5.30 \\
Left hippocampus anterior part & 141 & 30 & -6 & -20 & 4.86 \\
Left medial superior frontal (BA10) & 223 & -4 & 62 & 32 & 4.63 \\
Right middle temporal pole (BA38) & 102 & 50 & 12 & -24 & 4.48 \\
Left inferior frontal (BA45) & 182 & -54 & 34 & 12 & 4.27 \\
Right cerebellum crus 1 & 69 & 30 & -78 & -28 & 3.91 \\
\hline
\end{tabular}

Table 2: Brain regions activated during the familiarity experiment. $\mathrm{x}, \mathrm{y}, \mathrm{z}$ coordinates $(\mathrm{mm})$ are given in standard stereotactic MNI space. All regions listed are statistically significant at the $p<0.001$ level (uncorrected). 


\begin{tabular}{|c|c|c|c|c|c|}
\hline Contrast and anatomical location & $\begin{array}{l}\text { Cluster size } \\
\text { (in voxels) }\end{array}$ & $\mathbf{x}$ & $\mathbf{y}$ & $\mathbf{z}$ & Z score \\
\hline \multicolumn{6}{|l|}{ Musical Familiarity } \\
\hline Left inferior frontal (BA45) & 8947 & -52 & 30 & 10 & 5.72 \\
\hline $\begin{array}{l}\text { Right medial superior frontal and cingulate } \\
\text { (BA8) }\end{array}$ & 2553 & 8 & 18 & 26 & 5.61 \\
\hline Right inferior frontal (BA45) & 644 & 60 & 32 & 14 & 4.60 \\
\hline Right middle temporal (BA20) & 134 & 54 & -26 & -12 & 4.46 \\
\hline Left medial superior frontal (BA10) & 86 & -12 & 64 & 12 & 4.43 \\
\hline Right inferior frontal (BA47) & 245 & 42 & 32 & -10 & 4.14 \\
\hline Right superior temporal pole (BA38) & 155 & 54 & 14 & -16 & 4.13 \\
\hline Left middle and inferior temporal (BA20/37) & 277 & -58 & -48 & -12 & 4.13 \\
\hline Right middle frontal (BA9) & 56 & 32 & 34 & 40 & 3.91 \\
\hline Right rectus (BA11) & 50 & 6 & 34 & -20 & 3.69 \\
\hline \multicolumn{6}{|l|}{ Verbal Familiarity } \\
\hline Left inferior temporal (BA20) & 181 & -58 & -42 & -16 & 4.88 \\
\hline Left putamen & 581 & -32 & -10 & -6 & 4.80 \\
\hline Left medial superior frontal (BA10) & 2006 & -4 & 66 & 10 & 4.76 \\
\hline Right middle cingulate & 1088 & 6 & -18 & 34 & 4.74 \\
\hline Left inferior and middle frontal (BA47/46) & 208 & -34 & 40 & 6 & 4.68 \\
\hline Left middle frontal (BA8) & 426 & -24 & 16 & 64 & 4.44 \\
\hline Right hippocampus posterior part & 51 & 28 & -34 & -4 & 4.38 \\
\hline Right middle temporal (BA21) & 174 & 64 & 0 & -26 & 4.36 \\
\hline Left middle frontal (BA11) & 96 & -28 & 60 & -14 & 4.30 \\
\hline Left parahippocampal cortex & 386 & -14 & -30 & -8 & 4.19 \\
\hline Right middle frontal (BA8) & 152 & 34 & 22 & 50 & 4.17 \\
\hline Right cerebellum crus 1 & 341 & 48 & -60 & -24 & 4.06 \\
\hline Left olfactory (BA47) & 66 & -18 & 8 & -18 & 3.90 \\
\hline Left inferior temporal (BA20) & 77 & -60 & -12 & -24 & 3.89 \\
\hline Right inferior frontal (BA45) & 56 & 50 & 42 & 2 & 3.70 \\
\hline
\end{tabular}

Table 3: Activated brain regions representing the effect of familiarity of verbal and musical material. $\mathrm{x}, \mathrm{y}, \mathrm{z}$ coordinates $(\mathrm{mm})$ are given in standard stereotactic MNI space. All regions listed are statistically significant at the $p<0.001$ level (uncorrected). 


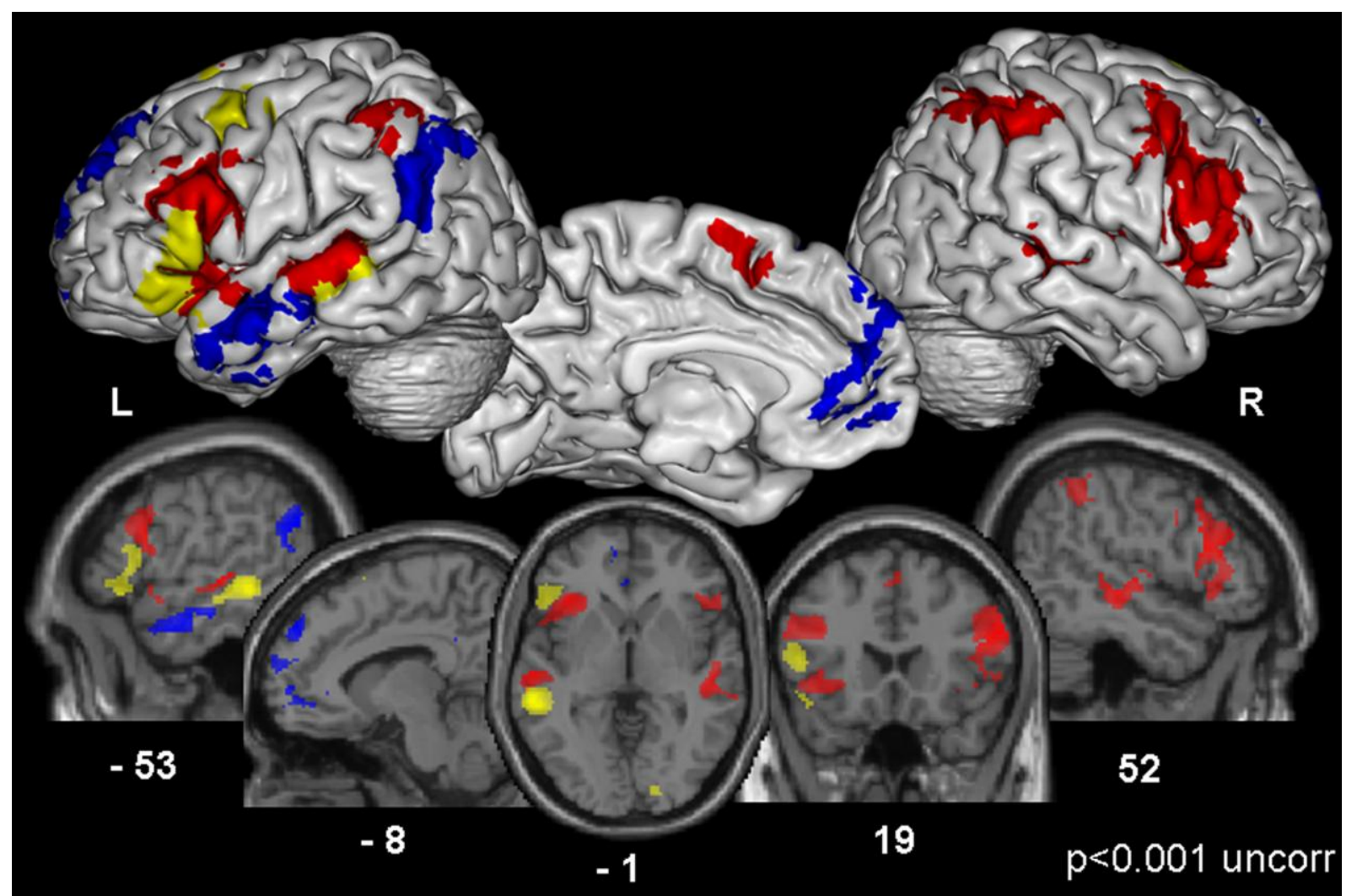

Figure 1: Brain areas activated during the congruence experiment: activation in the musical semantic contrast (in red), verbal semantic contrast (in blue) and conjunction analysis (in yellow) of musical semantic versus musical reference and verbal semantic versus verbal reference. Contrasts are displayed at $p<0.001$ (uncorrected) and superimposed onto an MNI template brain using Anatomist software (www.brainvisa.info). 


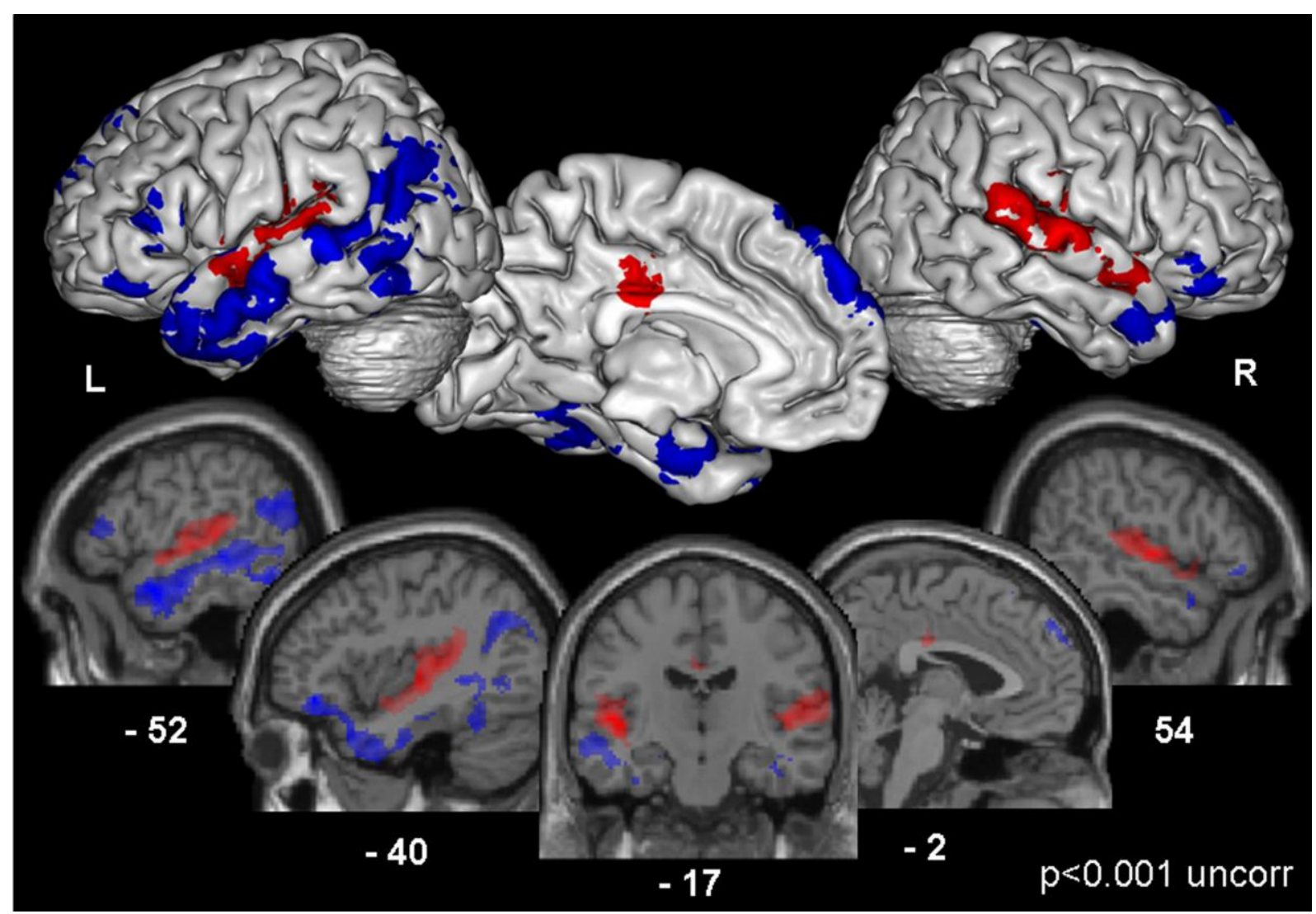

Figure 2: Brain areas activated during the familiarity experiment: activation in the musical contrast (in red) and verbal contrast (in blue). Contrasts are displayed at $p<0.001$ (uncorrected) and superimposed onto an MNI template brain using Anatomist software (www.brainvisa.info). 


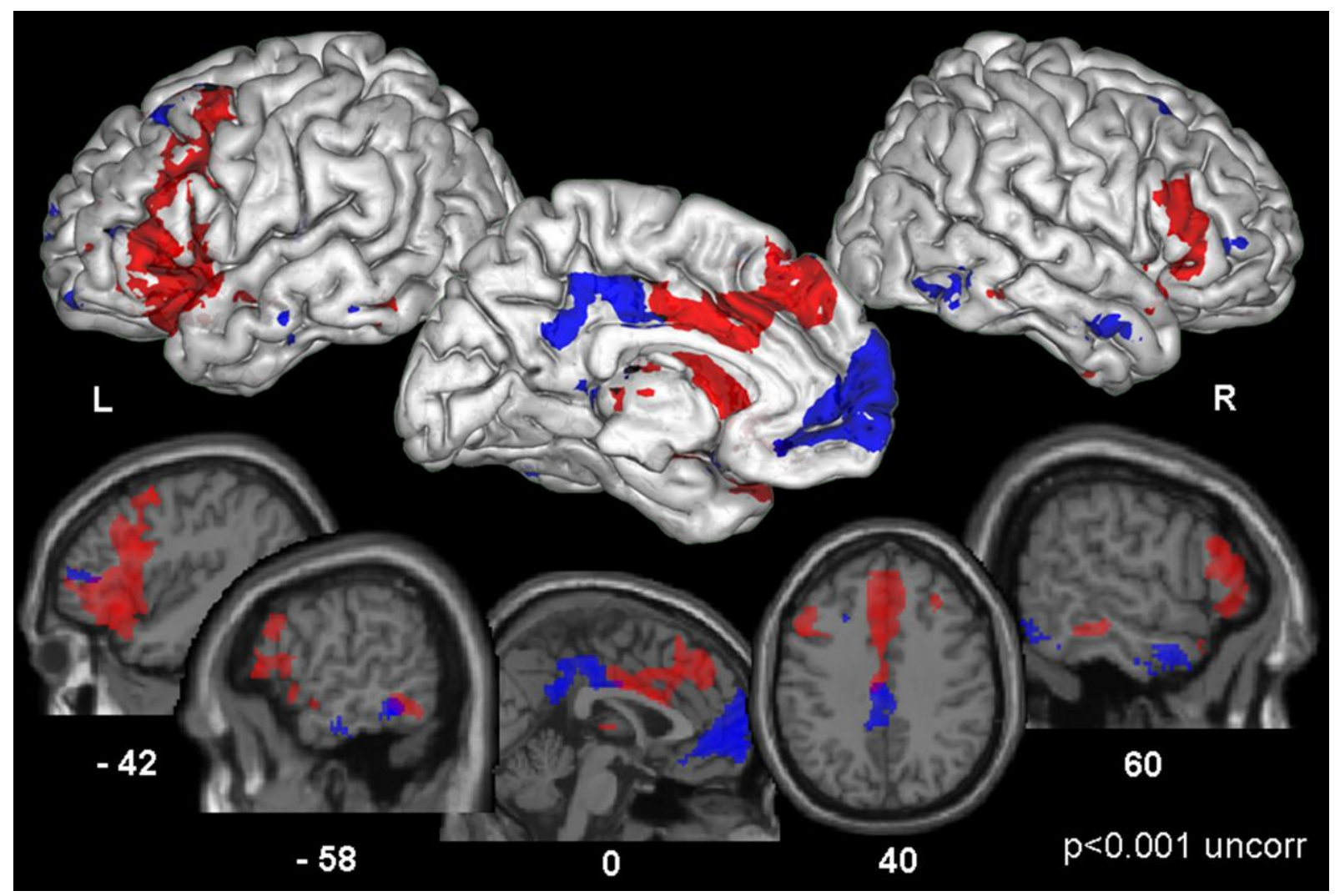

Figure 3: The familiarity experiment: effect of familiarity of music (in red) and language (in blue). Contrasts are displayed at $p<0.001$ (uncorrected) and superimposed onto an MNI template brain using Anatomist software (www.brainvisa.info). 